\title{
COLOUR IMAGE INTERPOLATION FOR HIGH RESOLUTION ACQUISITION AND DISPLAY DEVICES
}

\author{
Nicos Herodotou and Anastasios N. Venetsanopoulos \\ Department of Electrical and Computer Engineering \\ University of Toronto \\ 10 King's College Road, Toronto, Ontario \\ M5S 1A4, Canada
}

\begin{abstract}
Spatial interpolation is an important technique that is often used to perform an image zoom or to simply recover an original image from its downsampled version. The rapid advancements in hardware, both in acquisition and display devices, has made it possible to process high resolution digital colour images. However, the multichannel nature of colour images demands sophisticated signal processing algorithms that take into account the existing interchannel correlations when performing image expansion. Many conventional linear approaches exist. Nevertheless, these produce artifacts in the form of blockiness, jagged lines, and blurring in the interpolated image. In addition to this, these methods perform independently in each colour plane, thereby neglecting the colour component correlation. In this paper, a set of nonlinear Vector FIR-Median hybrid (VFMH) filters are applied to the interpolation problem. These schemes are based on the class of vector order statistic filters which have desirable properties, such as the preservation of edges and image details, and the preservation of interchannel correlations. Colour images are interpolated from their downsampled versions and all of the techniques are compared, both, quantitatively as well as qualitatively. Experimental results indicate that VFMH filters produce better quantitative, and visually pleasing results than linear techniques.
\end{abstract}

\section{INTRODUCTION}

The process of downsampling or decimation, is an effective way of reducing the spatial information in a digital image, whether it be for storage or transmission purposes. Conversely, the reverse procedure of this, referred to as interpolation or upsampling, is useful in recovering the original high resolution image from its downsampled version or for simply resizing or zooming a digital image. Decimation and interpolation schemes find their way into many practical applications, such as image/video zooming (for computer display devices or HDTV), optical scanners, high-resolution printers, video set-top boxes, image/video format conversion, and progressive image transmission systems. The PC and workstation market in particular, has seen tremendous growth over the past few years, with the addition of fast RISC-based CPU's, high speed I/O buses, hard disks and arrays, large screen monitors with increased scanning rates, and graphics accelerator cards with high speed graphics engines that support very high resolutions at 24 or more bits/pixel. These class of systems enable the acquisition, storage, processing, and display of high resolution true colour (i.e. 24 bits/pixel) images. With this advancement in hardware, many graphics board manufacturers have incorporated pixel zooming functions right into the graphics chips. However, the algorithms they employ are usually simple and linear based, which yield interpolated images of poor quality. Recent trends have also been to implement these features in hardware for on-the-fly image expansion. This is particularly important for digital video where large bandwidths are necessary. Video scaling eliminates this bandwidth bottleneck and permits real-time video playback at increased resolutions. Image acquisition devices, such as scanners, also utilize interpolation techniques in order to increase the optical resolution through software. Therefore, the need for robust, high quality interpolation algorithms has become increasingly important.

Many conventional interpolation techniques have been used to increase the spatial resolution of an image [1], [2], [3]. Some of these include pixel replication, bilinear interpolation, and spline based methods. However, these techniques often perform rather poorly in a subjective sense, as they tend to cause blurring or introduce artifacts in the form of jagged lines or blockiness in the interpolated image. This degradation in image quality is due to the deviations of the above linear filters from the ideal lowpass filter. Other statistical approaches based on Markov Random field models have also been successfully implemented in solving this problem [4], [5], [6]. These techniques require that parameters be estimated for the prescribed underlying image model. However, parameter estimation is a computationally demanding procedure that limits the usefullness of these algorithms for any real-time applications.

The conventional linear schemes described above are well established methods for univariate two-dimensional signals, such as grey level images. An extension of these techniques to multivariate data, such as colour images is not straightforward. Processing each colour component separately will fail to take into account the correlations between channels (i.e. colours). Moreover, the linear filters suffer from artifacts in the interpolated images. Recent work [7] has indicated that order statistic (OS) based nonlinear filters outperform their linear counterparts in output quality for this univariate case. In this paper, colour image interpolation is performed using a class of nonlinear filters based on vector OS filters. Due to the multichannel nature of colour, the multivariate samples are processed as vectors as opposed to component-wise scalars. Two different resampling (i.e. downsampling/upsampling) schemes are investigated here, the rectangular, and quincunx lattices. Colour images are 
interpolated from their downsampled images using various Vector FIR-Median hybrid (VFMH) filters, as well as the Vector Median (VMF), and are subsequently compared to the linear component-wise techniques. Experimental results are presented for both real and synthetic images.

\section{Order Statistic Filters}

Digital filters based on order statistics have found extensive applications in many areas of image processing, such as image analysis, restoration, enhancement, and noise removal [8]. The theoretical basis for this class of filters lies in the theory of robust statistics [9], [10]. Robust estimation aims to find the parameters that best fit the bulk of the data and to identify and reject the outlying observations. This is particularly important in image filtering, so that the edge information and image details are preserved while the noise is suppressed. This OS class includes many nonlinear filters, such as the median filter [11], [12], the $\alpha$-trimmed mean [13], and the median hybrid filter [14], to name a few. The appropriate filter is chosen according to the specific application and the characteristics of the problem at hand.

OS filters are based on the ordering of scalar data values for the univariate case. A generalization of the filtering process consists of the following three steps: i) windowing, ii) algebraic ordering, iii) and linear weighting. In an image $f$ (consisting of univariate values), a section of the image is first selected by a window $W$, yielding $W=\left\{f_{1}, f_{2}, \ldots, f_{n}\right\}$. The next step involves the algebraic ordering of these values, which results in the following $W=\left\{f_{(1)}, f_{(2)}, \ldots, f_{(n)}\right\}$, where the $f_{(i)}$, $i=1,2, \ldots, n$ are the ordered samples. Finally, these ordered values are weighted appropriately and summed. This process can be described mathematically by the expression $g_{i}=\sum_{j=1}^{n} a_{j} f_{(j)}$ where $f_{(j)}$ are the ordered samples, $a_{j}$ are the filter coefficients, and $g_{i}$ is the filter output. Different filters can be realized by properly selecting the filter coefficients. The following choices of coefficients result in the median, and the $\alpha$-trimmed mean filters, respectively

$$
\begin{aligned}
& a_{j}= \begin{cases}1 & j=\nu+1 \\
0 & j \neq \nu+1\end{cases} \\
& a_{j}=\left\{\begin{array}{cl}
\frac{1}{n(1-2 \alpha)} & j=\alpha n+1, \ldots, n-\alpha n \\
0 & \text { otherwise }
\end{array}\right.
\end{aligned}
$$

where $n=2 \nu+1$. A large number of filters can be realized with this structure. The performance of each filter in this OS class depends on several factors some of which include, image preservation characteristics, edge response, noise removal, and computational complexity. As mentioned earlier, the proper selection of a suitable filter depends on the specific image processing application.

\section{Median Hybrid Filters}

The median is the best known filter from the family of order statistics. Its widespread use is due to its simplicity, computational speed, edge preservation, and impulse removal properties. The median filter has been studied quite thoroughly and it exhibits very well known and desiralsle statistical [11] and deterministic (i.e. root signals which are invariant under median filtering) properties [12]. However, it has the undesirable effect of creating linear streaks or blotches in the filtered output image. Fine details, sharp corners, and narrow lines are also destroyed, because the ordering process in median filtering destroys any structural and spatial neighbourhood information. This is extremely undesirable in image interpolation, since fine details and image edges contain high frequency content that carry very important information for human visual perception. The median hybrid filter (FMH) has been shown to improve upon the image detail preservation properties of the median filter. In addition to this, the FMH filter performs well in attenuating noise, and preserving step edges.

Median hybrid filters are a combination of linear filters and median filters. Linear subfilters are used to take into account the spatial structure of the input image. The median hybrid filter is defined as follows

$$
g_{i}=\operatorname{Median}\left(\Phi_{1}\left(f_{i}\right), \ldots \Phi_{n}\left(f_{i}\right)\right)
$$

where $\Phi_{j}\left(f_{i}\right), j=1, \ldots n$ are linear FIR or IIR filters. The flexibility of this structure allows one to design the linear filters in such a way that fine horizontal, vertical, and/or diagonal lines can be preserved in an image. By using a small number of FIR subfilters of the averaging type, the number of sorting and multiply operations can also be greatly reduced [14]. This offers an improvement in speed over the median filter, while at the same time preserving the fine image details. Nieminen et al. report that the computation time of a multilevel detail preserving FMH filter is over two times less than that of a median filter, and over seven times less than a $\mathrm{K}$-nearest neighbour averaging filter in a $5 \times 5$ window [15].

An important property of FMH filters is the existence of root signals, where input sequences are invariant to repeated filtering operations. Roots signals can give an indication of the filter's ability to preserve fine lines and details when applied to an input image. In [15], a test image of a thin circular ring was generated and used to analyze the effects of filtering with various FMH filters, the median, and the $K$ averaging filter. When a very thin ring was used (i.e. width of 2 pixels), only an FMH filter with subfilters in all eight orientations did not change the input image upon filtering. All other filters altered the ring image. The median filter was not able to preserve the original image due to the small width of the ring while the averaging filter distorted the signal levels of the ring. Thus, this preservation property of the FMH filter is highly desirable.

The statistical properties of FMH filters have been analyzed for many important classes of input signals and noise distributions. A statistical analysis by computer simulation indicates that the FIR-Median hybrid filters are able to preserve edges in noisy images better than the median and the K-nearest neighbour averaging filter [15]. This edge preservation property is also important in retaining sharp, filtered output images. 
In image interpolation, four factors are of particular importance: i) edge preservation, ii) fine detail preservation, iii) unbiasedness (i.e. directional or illumination bias), iv) and computational complexity. FIR-Median filters perform strongly in all four of the above mentioned areas. [16] have included a comprehensive list of various nonlinear filters and their performance for various figures of merit. The tabulated results also indicate that the FMH filters are strong performers in all four areas indicated above, which are critical to the interpolation process.

\section{MUltichannel Data}

Multichannel or multispectral signal processing has been a rapidly growing area of interest recently, primarily due to the numerous applications. The advances in high resolution, true-colour graphics cards along with high scanning monitors, and state of the art active matrix TFT screens, have all demanded an increased attention in colour image processing techniques. However, the multichannel nature of colour adds an increased complexity when processing colour images. Data storage is multiplied by a factor of three and computational complexity also increases over the monochrome case. This is due to the fact that a colour image is a three channel, two dimensional signal (i.e. each pixel is a $3 \mathrm{D}$ vector composed of red, green, and blue additive components). In addition to this, a strong correlation exists between the different channels which suggests that a vector approach be taken in order to utilize the interchannel correlations [17]. Vector processing has been successfully applied recently, in many areas of image processing such as filtering [18], [19], enhancement [20], [21], edge detection [22], [23], and restoration [24], [25]. Transformation techniques such as the Karhunen-Loeve transform have also been used to decorrelate the three channels so that monochrome methods could be applied to each of the decoupled channels [26]. This is also a valid approach, however, multichannel signal processing techniques appear to be a more natural approach to the problem [25], [27]. Thus, univariate order statistic filters are extended to the case of multichannel data for colour image interpolation.

\section{Ordering of Multivariate Data}

In the univariate case, OS filters are based on the ordering of scalar data values and an extension to multivariate data is not straightforward. As mentioned earlier, colour images comprise an important class of multivariate signals and therefore an appropriate framework must be chosen for processing them (i.e. in order to take advantage of the interchannel correlations of the RGB colour planes). In the case of colour, each data sample is a vector value with red, green, and blue components. Let the set of $n$ vectors be within a window $W$, where $W=\left\{\mathbf{f}_{1}, \mathbf{f}_{2}, \ldots, \mathbf{f}_{n}\right\}$ and each vector, $\mathbf{f}_{i}=\left[R_{i}, G_{i}, B_{i}\right]$ be a point in the $\mathrm{RGB}$ colour space. The ordering of this multivariate data can be performed according to the following sub-ordering principles: Marginal ordering, reduced ordering, partial ordering, and conditional ordering [28]. In reduced ordering ( $R$ ordering), the vectors, $f_{i}$ are ranked according to some distance criteria. This reduces multivariate ranking to a simple scalar ranking operation on a set of distance values and retains the colour component correlations. For this reason, we restrict our attention to this ordering scheme. In R-ordering, the distance values are computed for each vector in the window as follows $d_{i}=\sum_{j=1}^{n}\left\|\mathbf{f}_{i}-\mathbf{f}_{j}\right\|$ $, i=1,2, \ldots, n$ using an appropriate vector norm [29]. Here we use the Euclidean distance due to its effectiveness and simplicity. Using this expression, one can compute an associated set of distances $\left\{d_{1}, d_{2}, \ldots, d_{n}\right\}$ for the set $\left\{\mathbf{f}_{1}, \mathbf{f}_{2}, \ldots, \mathbf{f}_{n}\right\}$, The scalar ranking of these distances yields the ranked vector set $\left\{f_{(1)}, f_{(2)}, \ldots f_{(n)}\right\}$, where the vector $\mathbf{f}_{(i)}$ is the $i^{t h}$ order statistic. This ranking process is the basis of the nonlinear Vector Median type filters described below.

\section{Vector Median Type Filters}

A natural extension of the median filter to the mutichannel case is the vector median (VMF). The VMF filter is known to exhibit properties similar to those of the scalar median, that is, the preservation of edges, the existence of root signals, and the suppression of impulsive noise [19]. Using the notation from the previous section, the vector median can be defined as follows

$$
\mathbf{f}_{V M}=\mathbf{f}_{(1)}=\operatorname{VM}\left\{\mathbf{f}_{1}, \mathbf{f}_{2}, \ldots, \mathbf{f}_{n}\right\}
$$

where VM is the vector median operation. The vector $\mathbf{f}_{V M}$ is the one whose distance to all other vectors is a minimum and is also contained in the set of vectors within the sliding window $W$

$$
\sum_{j=1}^{n}\left\|\mathbf{f}_{V M}-\mathbf{f}_{j}\right\|_{2} \leq \sum_{j=1}^{n}\left\|\mathbf{f}_{i}-\mathbf{f}_{j}\right\|_{2} \quad \forall i \in\{1, \ldots, n\}
$$

In a similar way, the FMH filters described earlier can be extended to the multichannel case using Vector FIRMedian hybrid (VFMH) filters. These are defined in an analogous manner

$$
\mathbf{f}_{V F M H}=\operatorname{VM}\left\{\boldsymbol{\Phi}_{1}, \mathbf{\Phi}_{2}, \ldots, \boldsymbol{\Phi}_{n}\right\}
$$

where $\boldsymbol{\Phi}_{i}$ are mutichannel, linear FIR filters. The VFMH filters are shown to have good edge preservation properties and good noise attenuation [30]. Once again, the flexibility of the linear FIR substructures allows one to reduce the computation time (by choosing a small number of linear subfilters), and preserve fine details of the image by selecting the $\boldsymbol{\Phi}_{i}$ appropriately. Furthermore, the VFMH filters preserve the edge shape and location for each signal component simultaneously by operating vectorially and utilizing the interchannel correlations. Component-wise filtering on the other hand, may cause edge shifts (i.e. known as edge jitter) in the different channels which may result in new, unwanted colours in transition areas where the original colour is rapidly changing. In [30], the VFMH filter is shown to outperform the component-wise FMH filter near a simulated step edge. Clearly, the VFMH filters described above, possess many desirable properties that make them suitable for image interpolation. 


\section{Rectangular and Quincunx Resampled LATTICES}

The interpolation of a one-dimensional signal by a factor of $L$ requires that for each known sample value, $L-1$ new samples be determined and inserted in the sequence. The placement of these newly determined values is straightforward in the $1-\mathrm{D}$ case, however, in the two-dimensional case of images, the spatial arrangement of these values can be quite arbitrary. This arrangement of pixel values forms a constellation or lattice of points. Two practical cases are examined here, that of rectangular and quincunx lattices. In this paper, the original image is decimated and then interpolated back to its original size, so that quantitative as well as qualitative comparisons can be made. This procedure is outlined below.

In two-dimensional decimation of images, a subset of the original 2-D sequence is retained and a sub-lattice of sampled points is formed. This lattice can be determined from the expression $g[\mathbf{n}]=f[\mathbf{M n}]$ for an $\mathbf{M}$-fold decimation process, where $\mathbf{M}$ is a $2 \times 2$ nonsingular matrix of integers, $\mathbf{n}=\left(n_{1}, n_{2}\right)\left(n_{1}\right.$, and $n_{2}$ are the horizontal and vertical spatial indices, respectively, in the $2-\mathrm{D}$ integer plane), $f[]$ is the original image, and $g[]$ is the output or subsampled set of points [31]. A number of different lattices can be generated by properly choosing the matrix M. The decimation ratio, or equivalently the compression ratio is found by taking the absolute value of the determinant of the matrix $\mathbf{M}$ (i.e. | det $\mathbf{M} \mid$ ). The rectangular and quincunx subsampled lattices are shown in Figure 1 below. If the $5 \times 5$ sample of points in the figure represent a particular section of an image, then only the points $\mathrm{X}$ are retained from the decimation process in each of the schemes mentioned above. Subsequent interpolation of the image from its decimated version requires that the missing pixels $Y$, and $\mathrm{Z}$ be determined, so that the regenerated image is as close as possible to the original. In the case of linear interpolation, the missing pixels are replaced with zeros and then this zero-interlaced image is subsequently lowpass filtered.

\begin{tabular}{|c|c|c|c|c|c|}
\hline \multirow{5}{*}{ a) } & $\mathrm{X}$ & $\mathrm{Y}$ & $\mathrm{X}$ & $\mathrm{Y}$ & $\mathrm{X}$ \\
\hline & $Y$ & $\mathrm{Z}$ & $\mathrm{Y}$ & $\mathrm{Z}$ & $\mathrm{Y}$ \\
\hline & $X$ & $\mathrm{Y}$ & $X$ & $Y$ & $\mathrm{X}$ \\
\hline & $\mathrm{Y}$ & $\mathrm{Z}$ & $\mathrm{Y}$ & $\mathrm{Z}$ & $Y$ \\
\hline & $X$ & $\mathrm{Y}$ & $X$ & $\mathrm{Y}$ & $X$ \\
\hline \multirow{5}{*}{ b) } & $\mathrm{X}$ & $\mathrm{Y}$ & $X$ & $\mathrm{Y}$ & $X$ \\
\hline & $Y$ & $X$ & $Y$ & $X$ & $Y$ \\
\hline & $\mathrm{X}$ & $Y$ & $\mathrm{X}$ & $\mathrm{Y}$ & $X$ \\
\hline & $\mathrm{Y}$ & $X$ & $\mathrm{Y}$ & $X$ & $\mathrm{Y}$ \\
\hline & $\mathrm{X}$ & $Y$ & $\mathrm{X}$ & $Y$ & $\mathrm{X}$ \\
\hline
\end{tabular}

Figure 1. a) Rectangular decimation b) Quincunx decimation.

\section{IMAGE INTERPOLATION USING VECTOR-FIR Median Hybrid Filters}

Several VFMH filters were used for each of the decimation schemes described previously. In rectangular decima- tion, the pixels $\mathrm{Y}$, and $\mathrm{Z}$ must be interpolated using the developed nonlinear filters. A subsection of the rectangular lattice of Figure 1a) is shown here to illustrate the six different VFMH filtering schemes that were implemented.

$$
\begin{array}{llll} 
& \mathrm{X}_{1} & \mathrm{Y}_{1} & \mathrm{X}_{2} \\
\mathrm{Z}_{2} & \mathrm{Y}_{2} & \mathrm{Z}_{1} & \mathrm{Y}_{3} \\
& \mathrm{X}_{3} & \mathrm{Y}_{4} & \mathrm{X}_{4}
\end{array}
$$

1. $\mathrm{VFMH}_{R 1}$ :

$$
\begin{aligned}
& \mathrm{Z} \text { pixels } \Rightarrow \\
& \mathrm{Z}_{1}=\mathrm{VM}\left\{\mathrm{X}_{1}, \mathrm{X}_{2}, \mathrm{X}_{3}, \mathrm{X}_{4}, \mathrm{X}_{\text {FIR } 1}\right\} \\
& \mathrm{Y} \text { pixels } \Rightarrow \\
& \mathrm{Y}_{1}=\left(\mathrm{X}_{1}+\mathrm{X}_{2}\right) / 2, \quad \mathrm{Y}_{2}=\left(\mathrm{X}_{1}+\mathrm{X}_{3}\right) / 2 \\
& \mathrm{Y}_{3}=\left(\mathrm{X}_{2}+\mathrm{X}_{4}\right) / 2, \quad \mathrm{Y}_{4}=\left(\mathrm{X}_{3}+\mathrm{X}_{4}\right) / 2
\end{aligned}
$$

2. $\mathrm{VFMH}_{R 2}$ :

$$
\begin{aligned}
& \mathrm{Z} \text { pixels } \Rightarrow \\
& \mathrm{Z}_{1}=\mathrm{VM}\left\{\mathrm{X}_{1}, \mathrm{X}_{2}, \mathrm{X}_{3}, \mathrm{X}_{4}, \mathrm{X}_{\text {FIR1 }}\right\} \\
& \mathrm{Y} \text { pixels } \Rightarrow \\
& \mathrm{Y}_{2}=\mathrm{VM}\left\{\mathrm{X}_{1}, \mathrm{Z}_{1}, \mathrm{Z}_{2}, \mathrm{X}_{3}, \mathrm{X}_{\text {FIR2 }}\right\}
\end{aligned}
$$

\section{3. $\mathrm{VFMH}_{R 3}$ :}

$$
\begin{aligned}
& \mathrm{Z} \text { pixels } \Rightarrow \\
& \mathrm{Z}_{1}=\mathrm{VM}\left\{\mathrm{X}_{1}, \mathrm{X}_{2}, \mathrm{X}_{3}, \mathrm{X}_{4}, \mathrm{X}_{F I R 1}\right\} \\
& \mathrm{Y} \text { pixels } \Rightarrow \\
& \mathrm{Y}_{2}=\mathrm{VM}\left\{\mathrm{X}_{1}, \mathrm{Z}_{1}, \mathrm{Z}_{2}, \mathrm{X}_{3}, \mathrm{X}_{H}, \mathrm{X}_{V}, \mathrm{X}_{\text {FIR } 2}\right\}
\end{aligned}
$$

4. $\mathrm{VMF}_{R}$ :

$$
\begin{aligned}
& Z \text { pixels } \Rightarrow \\
& Z_{1}=V M\left\{X_{1}, X_{2}, X_{3}, X_{4}\right\} \\
& Y \text { pixels } \Rightarrow \\
& Y_{2}=V M\left\{X_{1}, Z_{1}, Z_{2}, X_{3}\right\}
\end{aligned}
$$

An additional three filters, $\mathrm{VFMH}_{R 4}, \mathrm{VFMH}_{R 5}$, and VFMH $_{R 6}$, can also be realized by replacing the expression for $\mathrm{Z}_{1}$, in $\mathrm{VFMH}_{R 1}$, VFMH $\mathrm{VM}_{R 2}$, and $V F \mathrm{VH}_{R 3}$ above, by $\mathrm{Z}_{1}$ $=\mathrm{VM}\left\{\mathrm{X}_{1}, \mathrm{X}_{2}, \mathrm{X}_{3}, \mathrm{X}_{4}, \mathrm{X}_{D 1}, \mathrm{X}_{D 2}, \mathrm{X}_{F I R 1}\right\}$, respectively. In the expressions above, $\mathrm{X}_{D 1}=\left(\mathrm{X}_{1}+\mathrm{X}_{4}\right) / 2, \mathrm{X}_{D 2}=\left(\mathrm{X}_{2}+\right.$ $\left.\mathrm{X}_{3}\right) / 2, \quad \mathrm{X}_{H}=\left(\mathrm{Z}_{1}+\mathrm{Z}_{2}\right) / 2, \quad \mathrm{X}_{V}=\left(\mathrm{X}_{1}+\mathrm{X}_{3}\right) / 2$ and $\mathrm{X}_{F I R 1}, \mathrm{X}_{F I R 2}$, are 12 point, lowpass, FIR filters. One may also note that the filter structures of methods $2,3,5$, and 6 are recursive. The Vector Median filter $\left(\mathrm{VMF}_{R}\right)$ was also applied for comparison purposes. However, median 
filtering as was mentioned earlier, tends to destroy fine image details [8], and thus, the median hybrid filters should be preferred. All of the $Y$, and $Z$ pixels in Figure 1a) can be computed by implementing each of the filter schemes in one pass over the image. Similar structures have been applied successfully using the scalar median for the case of univariate data [7].

In the quincunx subsampled lattice of Figure 1b), five nonlinear interpolators were examined. The filter definitions below refer to the following $3 \times 3$ subsection of this latitice.

$\begin{array}{ccc}Y & X_{1} & Y \\ X_{2} & Y_{1} & X_{3} \\ Y & X_{4} & Y\end{array}$

\section{1. $\mathrm{VFMH}_{Q 1}$ :}

$\mathrm{Y}$ pixels $\Rightarrow$

$\mathrm{Y}_{1}=\mathrm{VM}\left\{\mathrm{X}_{1}, \mathrm{X}_{2}, \mathrm{X}_{3}, \mathrm{X}_{4}, \mathrm{X}_{A V E}\right\}$

2. $\mathrm{VFMH}_{Q 2}$ :

$Y$ pixels $\Rightarrow$

$\mathrm{Y}_{1}=\mathrm{VM}\left\{\mathrm{X}_{1}, \mathrm{X}_{2}, \mathrm{X}_{3}, \mathrm{X}_{4}, \mathrm{X}_{F I R}\right\}$

3. $\mathrm{VFMH}_{Q 3}$ :

$\mathrm{Y}$ pixels $\Rightarrow$

$\mathrm{Y}_{1}=\mathrm{VM}\left\{\mathrm{X}_{1}, \mathrm{X}_{2}, \mathrm{X}_{3}, \mathrm{X}_{4}, \mathrm{X}_{H}, \mathrm{X}_{V}, \mathrm{X}_{F I R}\right\}$

4. VTMH $_{Q 4}$ :

$Y$ pixels $\Rightarrow$

$\mathrm{Y}_{1}=\mathrm{VM}\left\{\mathrm{X}_{1}, \mathrm{X}_{2}, \mathrm{X}_{3}, \mathrm{X}_{4}, \mathrm{X}_{H F I R}, \mathrm{X}_{V F I R}, \mathrm{X}_{F I R}\right\}$

5. $\mathrm{VMF}_{Q}:$

$\mathrm{Y}$ pixels $\Rightarrow$

$\mathrm{Y}_{1}=\mathrm{VM}\left\{\mathrm{X}_{1}, \mathrm{X}_{2}, \mathrm{X}_{3}, \mathrm{X}_{4}\right\}$

where $\mathrm{X}_{A V E}=\left(\mathrm{X}_{1}+\mathrm{X}_{2}+\mathrm{X}_{3}+\mathrm{X}_{4}\right) / 4, \mathrm{X}_{H}=\left(\mathrm{X}_{2}+\mathrm{X}_{3}\right) / 2$, $\mathrm{X}_{V}=\left(\mathrm{X}_{1}+\mathrm{X}_{4}\right) / 2$ and $\mathrm{X}_{H F I R}, \mathrm{X}_{V F I R}$, are 4 point horizontal and vertical FIR filters, respectively, and $\mathrm{X}_{F I R}$, is a 12 point FIR filter. All of the $\mathrm{Y}$ pixels can be computed in a similar fashion to $Y_{1}$. The different FIR subfilters above are direction sensitive (i.e. the masks of the filter are in various orientations - horizontal, vertical, diagonal, and/or lowpass) and are chosen in order to preserve the fine details of the image.

\section{EXPERIMENTAL RESULtS.}

The nonlinear interpolating filters outlined in the previous section were applied to two different images, a $512 \times 480$ real colour image of "Lena", and a $512 \times 480$ synthetic image composed of several colours in different orientations (vertical, horizontal, and diagonal). Three linear schemes were also used for comparison purposes. In the rectangular lattice, a resampling (downsampling/upsampling) ratio of 16 was used while in quincunx, a ratio of 2 was employed. A summary of the results are tabulated on the following page. The mean square error (MSE) and mean absolute error (MAE) criteria were used to compare the results quantitatively and are defined as follows

$$
\begin{aligned}
\mathrm{MSE} & =\frac{1}{M N} \sum_{i=0}^{M} \sum_{j=0}^{N}\left\|\mathbf{f}_{i j}-\widehat{\mathbf{f}}_{i j}\right\|_{2}^{2} \\
\mathrm{MAE} & =\frac{1}{M N} \sum_{i=0}^{M} \sum_{j=0}^{N}\left\|\mathbf{f}_{i j}-\widehat{\mathbf{f}}_{i j}\right\|_{1}
\end{aligned}
$$

where $M, N$ are the image dimensions, $\mathbf{f}_{i j}$ is the vector value of the pixel $(i, j)$ of the original image, $\widehat{\mathbf{f}}_{i j}$ is the vector value of the pixel $(i, j)$ of the interpolated image, and \|\|$_{2},\|\|_{1}$, are the $L_{2}$, and $L_{1}$ vector norms, respectively. The tabulated results indicate that most of the nonlinear interpolation methods outperform their linear counterparts. The results are fairly consistent in each case, that is, overall the $\mathrm{VFMH}_{R 5}$, and $\mathrm{VFMH}_{Q 3}$, methods appear to be the best in the two resampling schemes. The bilinear method seems to perform better than the other linear schemes. The nonlinear interpolation techniques also perform much better than the conventional linear ones from a perceptual point of view. The interpolated images derived from the linear schemes have blocky, jagged lines, and are blurred, while the nonlinear methods appear to somewhat suppress these artifacts, preserve the edges, and retain the image details better. Figures 2, and 3 illustrate the output obtained from the bilinear method and the VFMH $V_{R 5}$ algorithm for the case of rectangular resampling. In the bilinear case, the aliasing effects (i.e. the jagged lines) are much more apparent and the edges are also blurred. This is quite evident in the synthetic image of Figure 3a), where the jagged lines are quite pronounced in the diagonal stripes of the image. The $\mathrm{VFMH}_{R 5}$ eliminates this problem as shown in Figure $3 b$ ), and performs quite well near step edges, and preserves the interchannel colour correlations. The strong performance of the $\mathrm{VFMH}_{R 5}$ filter can be attributed to: i) its FIR subfilters which consist of lowpass filters, as well as masks in the diagonal orientations, ii) and its processing of the multichannel data in a vectorial fashion which preserves colour correlations near signal transitions. One may also note a performance improvement in going from method 1 to 4,2 to 5 , and 3 to 6. Once again, this is due to the addition of the diagonally oriented FIR subfilters in the determination of the pixels, $Z_{i}$. The two linear schemes, other than the bilinear method, performed even poorer and resulted in blocky interpolated images when pixel replication was used, and very blurred outputs when the cubic B-spline method was applied. The vector median filter also did not perform well as it lost quite a bit of image detail, as expected. Consistent results were obtained for both images, and resampling 
TABLE I

A QUANTITATIVE COMPARISON OF THE DIFFERENT INTERPOLATION METHODS USING RECTANGULAR DECIMATION

\begin{tabular}{|l|c|c|c|c|}
\hline \multicolumn{4}{|c|}{ RECTANGULAR RESAMPLING } \\
\hline \multirow{2}{*}{ Method } & \multicolumn{2}{|c|}{ "Lena" } & \multicolumn{2}{c|}{ Synthetic Image } \\
\cline { 2 - 5 } & MSE & MAE & MSE & MAE \\
\hline Pixel Replication & 1098.2 & 30.44 & 1377.3 & 9.94 \\
Bilinear & 363.5 & 19.16 & 743.8 & 10.25 \\
Cubic B-spline & 423.6 & 21.72 & 833.1 & 10.25 \\
VFMH $_{R 1}$ & 360.9 & 18.95 & 743.4 & 9.21 \\
VFMH $_{R 2}$ & 364.1 & 19.15 & 760.9 & 8.84 \\
VFMH $_{R 3}$ & 408.8 & 20.31 & 771.7 & 8.70 \\
VFMH $_{R 4}$ & 351.3 & 18.71 & 726.1 & 9.58 \\
VFMH $_{R 5}$ & 346.9 & 18.58 & 726.9 & 9.58 \\
VFMH $_{R 6}$ & 359.2 & 18.90 & 729.8 & 9.44 \\
VMF $_{R}$ & 381.9 & 19.38 & 932.4 & 8.33 \\
\hline
\end{tabular}

TABLE II

A QUANTITATIVE COMPARISON OF THE DIFFERENT INTERPOLATION METHODS USING QUINCUNX DECIMATION

\begin{tabular}{|l|c|c|c|c|}
\hline \multicolumn{4}{|c|}{ QUINCUNX RESAMPLING } \\
\hline \multirow{2}{*}{ Method } & \multicolumn{2}{|c|}{ "Lena" } & \multicolumn{2}{c|}{ Synthetic Image } \\
\cline { 2 - 5 } & MSE & MAE & MSE & MAE \\
\hline Pixel Replication & 172.71 & 9.70 & 142.21 & 1.01 \\
Bilinear & 53.57 & 6.01 & 105.42 & 2.19 \\
VFMH $_{Q 1}$ & 51.97 & 5.93 & 45.74 & 0.67 \\
VFMH $_{Q 2}$ & 54.26 & 6.26 & 45.37 & 0.66 \\
VFMH $_{Q 3}$ & 47.83 & 5.85 & 45.05 & 0.66 \\
VFMH $_{Q 4}$ & 57.29 & 6.46 & 45.58 & 0.67 \\
VMF $_{Q}$ & 89.31 & 7.27 & 140.93 & 1.00 \\
\hline
\end{tabular}

schemes. The computational complexity of the $\mathrm{VFMH}_{R 5}$ filter is amongst the highest of the nonlinear methods, however, it is on the same order as the cubic B-spline method. This increased complexity comes at the expense of having a larger number of subfilters in the window of the vector median operation. The nonlinear interpolators are therefore, quite feasible for implementation. These structures can be designed in hardware as one functional unit of the graphics chip or as a separate ASIC that can provide hardware assisted interpolation on a board level solution (i.e. capture and/or video display device, HDTV applications, or multimedia set-top boxes for video on demand).

\section{Conclusions}

Spatial interpolation of colour images was investigated in this paper for applications in high resolution acquisition and display devices. Nonlinear filtering techniques based on vector order statistics were examined for this purpose. Vector FIR-Median hybrid filters were selected from this OS class of filters due to their robustness, preservation of edge information and image details, and their ability to exploit the existing correlation between the RGB colour planes. Several VFMH filters were implemented and compared to the conventional linear techniques using both real and synthetic images. The interpolated images were determined from their downsampled versions for two different decimation schemes, that of rectangular and quincunx decimation. Experimental results indicated that the VFMH filters performed better, both, quantitatively, and aesthetically than the linear methods. The former filtering techniques reduce the eflects of aliasing (blockiness, jagged lines, and blurring), preserve the step edges and image details better than their linear counterparts, and retain the correlations between colours by operating vectorially. In addition to this, the computational complexity of these nonlinear filters is on the same order as their linear counterparts which makes them attractive for implementation in hardware.

\section{REFFRFNCES}

[1] A.J. Parker, R.V. Kenyon, D.E. Troxel, 'Comparison of Interpolating Methods for Image Resampling', IEEE Trans. on Medical Imaging, vol. 2, no. 1, pp. 31-39, March 1983.

[2] H.H. Hon, H.C. Andrews, 'Cubic Splines for Image Interpolation and Digital Filtering', IEEE Trans. on Acoustics, Speech, and Signal Processing, vol. 26, no. 6, pp. 508-517, Dec. 1978.

[3] R.G. Keys, 'Cubic Convolution Interpolation for Digital Image Processing', IEEE Trans. on Acoustics, Speech, and Signal Processing, vol. 29, no. 6, pp. 1153-1160, Dec. 1981.

[4] R.R. Schultz, R.L. Stevenson 'A Bayesian Approach to Image Expansion for Improved Definition', IEEE Trans. on Image Processing, vol. 3 ,no. 3, pp. 233-242, May 1994.

[5] S. Lakshmanan, A.K. Jain, Y. Zhong, 'Multi-Resolution Image Representation Using Markov Random Fields', IEFE Proc. ICIP, Vol. I, pp. 855-860, 1994.

[6] N. Herodotou, L. Onural, A.N. Venetsanopoulos, 'Image Interpolation Using a Simple Gibbs Random Field Model', ICIP 95, Washington D.C., Oct. 1995.

[7] B. Zeng, A.N. Venetsanopoulos, 'A Comparative Study of Several Nonlinear Image Interpolation Schemes', Proceedings SPIE: Visual Communications and Image Processing, Vol. 1818, pp. 21-29, Nov. 1992.

[8] I. Pitas, A.N. Venetsanopoulos, 'Nonlinear Digital Filters', Kluwer Academic Publishers, Massachusetts, 1990.

[9] H.A. David, 'Order Statistics', John Wiley, New York, 1981.

[10] P.S. Huber, 'Robust Statistics', Joln Wiley, New York, 1981.

[11] B.I. Justusson, 'Median Filtering: Statistical Properties', TwoDimensional Digital Signal Processing II, T.S. Huang, Ed., Springer Verlag, New York, 1981.

[12] S.G. Tyan, 'Median Filtering: Deterministic Properties', TwoDimensional Digital Signal Processing II, T.S. Huang, Ed., Springer Verlag, New York, 1981.

[13] J.B. Bednar, T.L. Huang, 'Alpha-Trimmed Means and their Relationship to the Median Filters', IEEE Trans. on Acoustics, Speech, and Signal Processing, vol. 32, no. 1, pp. 145-153, Feb. 1984.

[14] P. Heinonen, Y. Neuvo, 'FIR-Median Hybrid Filters', IEEE Trans. on Acoustics, Speech, and Signal Processing, vol. 35, no. 6, pp. 832-838, June 1987.

[15] A. Nieminen, P. Heinonen, Y. Neuvo, 'A New Class of DetailPreserving Filters for Image Processing', IEEE Trans, on Pattern Analysis and Machine Intelligence, vol. 9, no. 1, Jan. 1987.

[16] I. Pitas, A.N. Venetsanopoulos, 'Order Statistics in Digital Image Processing', Proc. of the IEEE, vol. 80, no. 12, Dec. 1992.

[17] P.E. Trahanias, I. Pitas, A.N. Venetsanopoulos, 'Color Image Processing', Advances in 2D and 3D Digital Processing (Techniques and Applications), edited by C.T. Leondes, Academic Press, 1994. 
[18] P.E.: Trahanias, A.N. Venetsanopoulos, 'Vector Directional Filters - A New Class of Multicliamnel Image Processing Filters', IEEF, Trans. on Image Processing, vol. 2, no. 4, Oct. 1993.

[19] J. Astola, P. Haavisto, Y. Neuvo, 'Vector Median Filters', Proc. of the IEEE, vol. 78, no. 4, April 1990.

[20] R. Strickland, C. Kim, W. McDonnel, 'Digital Color Image Enhancement Based on the Saturation Component', Optical Engineering, vol. 26 , pp. 609-616, July 1987.

[21] P.E. Trahanias, A.N. Venetsanopoulos, 'Color Image Enhancement through 3D Histogram Equalization', Proc. 11th Int. Conf. Pattern Recognition, IEEE Computer Soc., The Hague, Netherlands, pp. 545-548, Aug. 1992.

[22] A. Cumani, 'Edge Detection in Multispectral Images', CVGIP: Giaphical Models and Image Processing, vol. 53, pp. 40-51, Janl. 1991.

[23] P. M. Thahanias, A.N. Venetsanopoulos, 'Color Edge Detection Using Vector Order Statistics', ILEE Trans. on Image Processing, vol. 2, no. 2, April 1993.

[24] N.P. Galatsanos, R.T. Chin, 'Digital Restoration of Multichannel Images', IEEF Trans, on Acoustics, Speech, and Signal Processing, vol. 37, pp. 415-421, March 1989.

[25] N.P Galatsanos, R.T. Chin, 'Restoration of Color Images by Multichannel Kalman Filtering', IEEE Trans. on Acoustics, Speech, and Signal Processing, vol. 39, pp. 2237-2252, Oct. 1991.

[26] B.R. Hunt, 'Karhunen-Loeve Multispectral Image Restoration, Part I: Theory', IEEE Trans. on Acoustics, Speech, and Signal Processing, vol. 32, no. 3, pp. 592-599, June 1984.

[27] G. Aggelopoulos, I. Pitas, 'Least-Squares Multichannel Filters in Color Image Restoration', Proc. European Conference on Circuit Theory and Design ECCTD89, Brighton, England, Sept. 1989.

[28] V. Barnett, 'The Ordering of Multivariate Data', Journal of the Royal Statistical Society A, Vol. 139, pt. 3, pp. 318-354, 1976.

[29] R.C. Hardie, G.R. Arce, 'Ranking in $\mathrm{R}^{p}$ and its Use in Multivariate Image Estimation', IEEE Trans. on Circuits and Systems for Video Technology, Vol. 1, No. 2, pp. 197-209, June 1991.

[30] P. Haavisto, P. Heinonez, Y. Neuvo, 'Vector FIR-Median Hybrid Filters for Multispectral Signals', Electronic Letters, vol. 24, no. 1, pp. 7-8, Jan. 1988.

[31] P.P. Vaidyanathan, 'Multirate Systems and Filter Banks', Prentice Hall Inc., New Jersey, 1993.

\section{BIOGRAPHIES}

Nicos Herodotou (S'85) received the B.A.Sc. in 1987, and the M.A.Sc. in 1990, both in Electrical Engineering from the University of Toronto. He has industry experience in designing analog filters for power line carrier transmission systems, and has consulted in projects for multimedia software development, and PC systems integration for image and video applications. He is a registered Professional Engineer in the province of Ontario (APEO - Association of Professional Engineers of Ontario) and is a member of the IEEE Signal Processing, and Circuits and Systems Societies. He is currently a Ph.D. candidate at the University of Toronto in the Department of Electrical and Computer Engineering. His current research interests include image and video processing, multidimensional/multichannel linear and nonlinear filtering, and image/video compression.

Anastasios N. Venetsanopoulos (S'66-M'69-SM'79F'88) received the B. Eng. degree from the National Technical University of Athens (NTU), Greece, in 1965, and the M.S., M. Phil., and Ph.D. degrees in Electrical Engineering from Yale University in 1966, 1968 and 1969 respectively. He joined the University of Toronto in September 1968, where he is a Professor in the Department of Electrical and Computer Engineering since 1981. He has served as Chairman of the Department of the Communications Group and Associate Chairman of the Department of Electrical Engineering. He was on research leave at the Federal University of Rio de Janeiro, the Imperial College of Science and Technology, the National Technical University of Athens, Swiss Federal Institute of Technology, and the University of Florence, and was Adjunct Professor at Concordia University. He has served as Lecturer in 130 short courses to industry and continuing education programs and as Consultant to several organizations; he is a contributor to twenty-four books, a co-author of Nonlinear Filters in Image Processing: Principles and Applications and Artificial Neural Networks: Learning Algorithms, Performance Evaluation and Applications, and has published over 490 papers on digital signal and image processing and digital communications. He has served as Chairman on numerous boards, councils and technical conference committees including IEEE committees such as the Toronto Section (1977-1979) and the IEEE Central Canada Council (1980-1982); he was President of the Canadian Society for Electrical Engineering and Vice President of the Engineering Institute of Canada (1983-1986). He has been a Guest Editor or Associate Editor for several IEEE journals and the Editor of the Canadian Electrical Engineering Journal (1981-1983). He is a member of the IEEE Communications, Circuits and Systems, Computer, and Signal Processing Societies, as well as a member of Sigma Xi, the technical Chamber of Greece, the European Association of Signal Processing, the Association of Professional Engineers of Ontario (APEO) and Greece. He was elected as a Fellow of the IEEE 'for contributions to digital signal and image processing', also Fellow of EIC and was awarded an Honourary Doctorate from the National Technical University of Athens, for his 'contribution to engineering' in October 1994. His present research interests include: Linear M-D and Nonlinear filters, processing of multispectral (colour) images and image sequences, telecommunications and image compression. In particular, the development of efficient techniques for multispectral image transmission, restoration, filtering and analysis. 


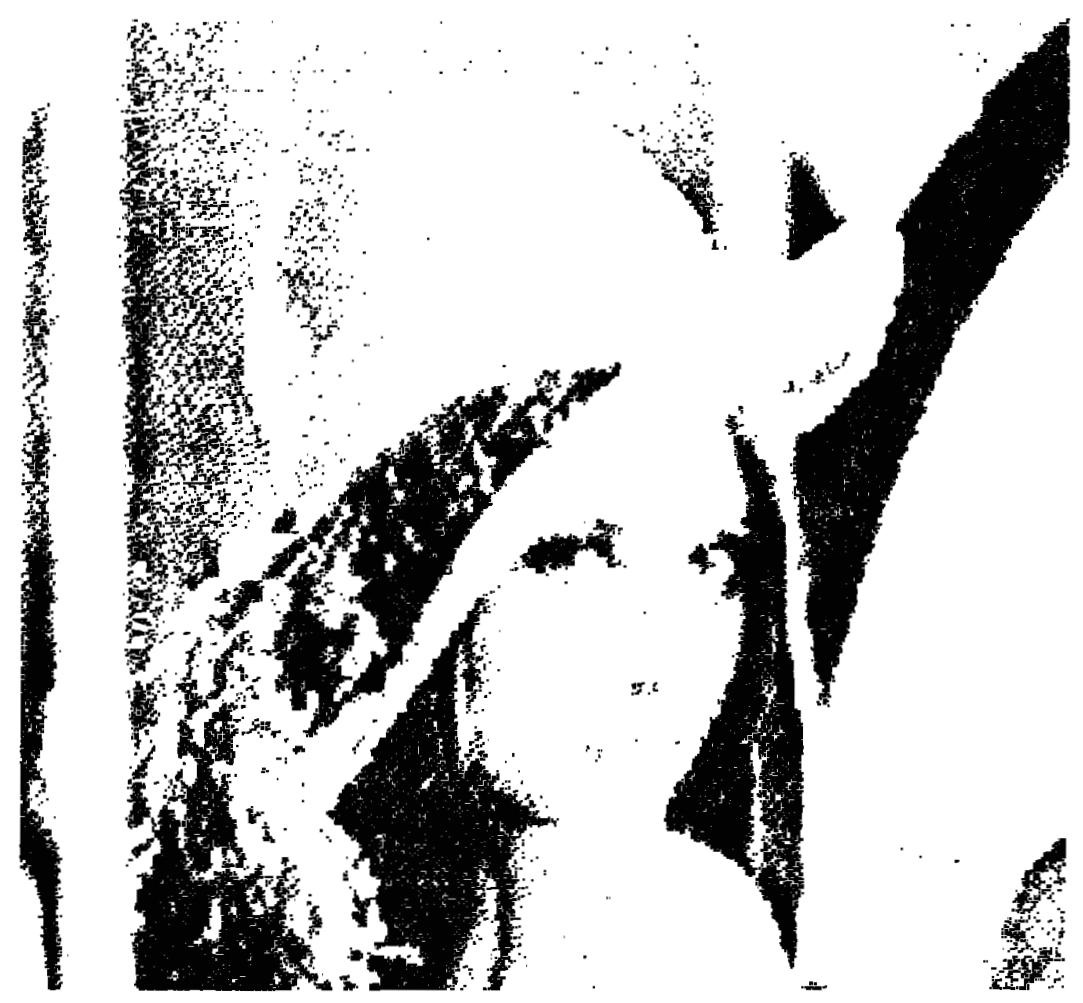

Figure 2a): Linear interpolation of "Lena" image using the bilinear method for rectangular resampling. A downsampling/upsampling factor of 16 was used (i.e. by 4 in each direction).

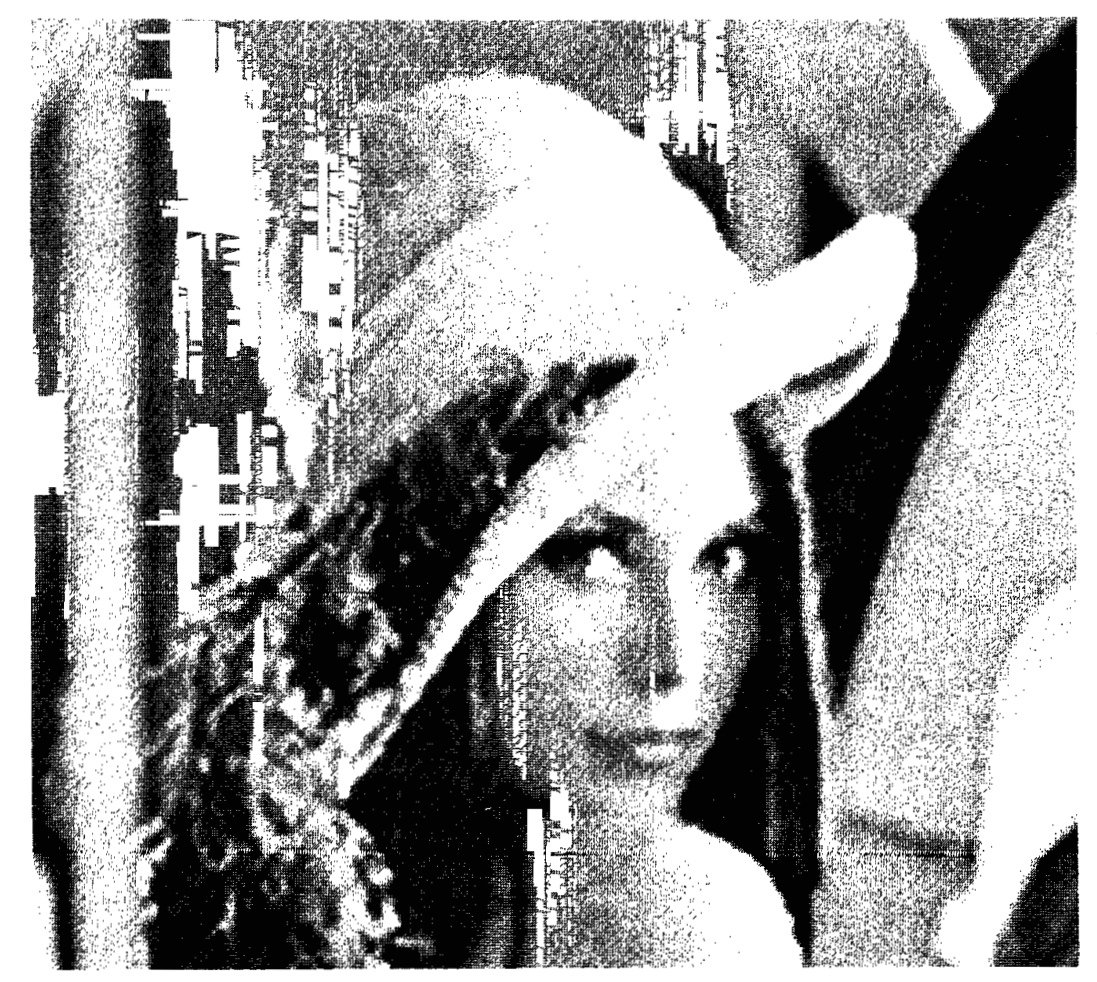

Figure 2b): Nonlinear interpolation of "Lena" image using the $\mathrm{VFMH}_{R s}$ method for rectangular resampling. A downsampling/upsampling factor of 16 was used (i.e. by 4 in each direction). 

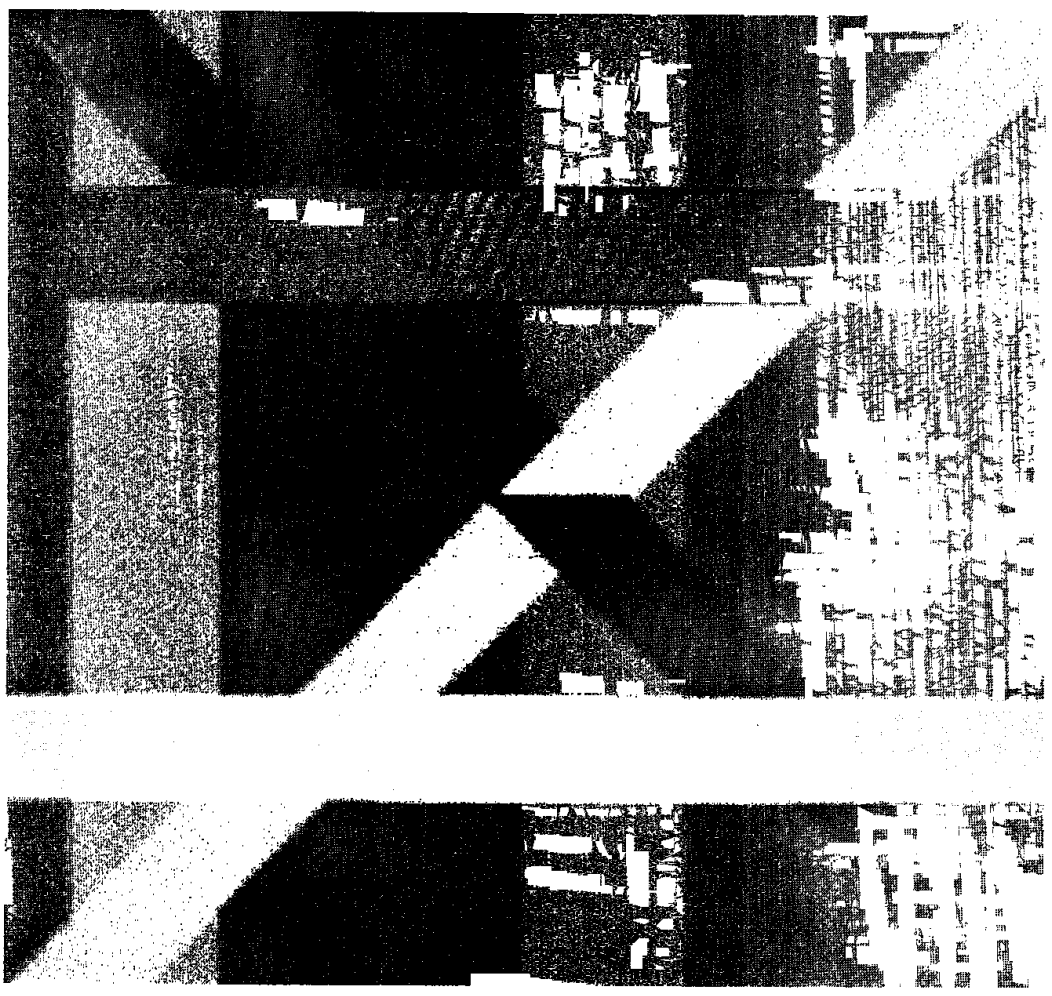

Figure 3a): Linear interpolation of synthetic image using the bilinear method for rectangular resampling. A downsampling/upsampling factor of 16 was used (i.e. by 4 in each direction).
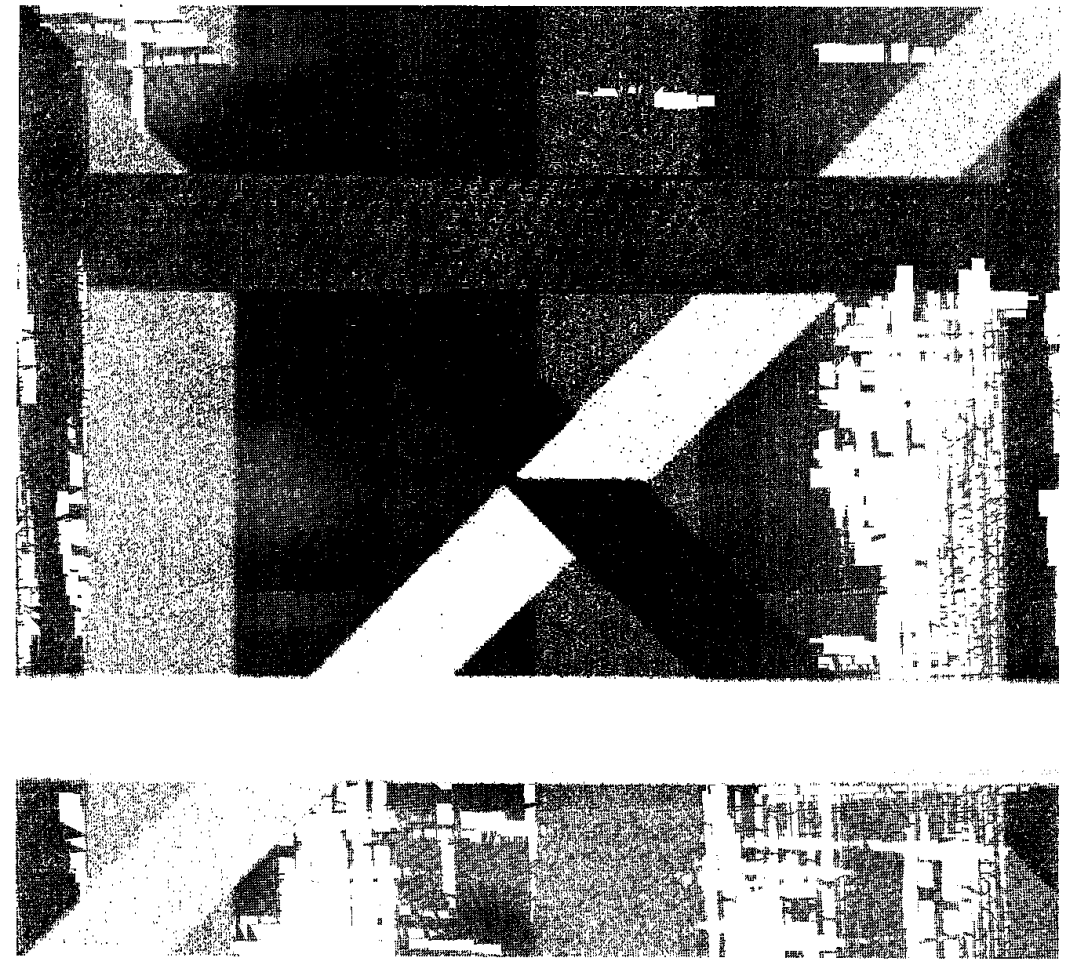

Figure 3b): Nonlinear interpolation of synthetic image using the $\mathrm{VFMH}_{R 5}$ method for rectangular resampling. A downsampling/upsampling factor of 16 was used (i.e. by 4 in each direction). 